Macedonian Pharmaceutical Bulletin, 66 (Suppl 1) 33 - 34 (2020)

Online ISSN 1857 - 8969

UDC: 616.345-009-083.2

DOI: 10.33320/maced.pharm.bull.2020.66.03.016

Short communication

\title{
Dietetic approach in patients with irritable bowel syndrome - a case report
}

\author{
Menka Andreska*, Bojana Janeku, Dafina Boshkoska, Elena Karabeleski, Suzana \\ Atanasovikj, Dragana Mladenovska, Aleksandra Kapedanovska Nestorovska, Tanja \\ Petreska Ivanovska, Kristina Mladenovska, Lidija Petrushevska-Tozi
}

Faculty of Pharmacy, Ss. Cyril and Methodius University, Mother Theresa No. 47, 1000 Skopje, N. Macedonia

\section{Introduction}

Irritable bowel syndrome (IBS) is a functional gastrointestinal disorder, with high prevalence of $7-21 \%$ globally. One of 10 people has IBS or some of the symptoms due to various factors. Different countries have different incidence rate - the average of the available statistics is $2-70 / 1000$ patients per year. It is a syndrome with a set of symptoms that can occur in different forms, intervals and intensity. IBS is a lifelong problem with a major impact on the quality of life of the patient, family and environment. Occurs more often in the female then male ( 2 of 3 cases are female). In terms of age, it usually occurs before the age of 50 (between 20-30 years), but there is no age limit as symptoms can be also encountered in children (McKenzie et al., 2016).

Symptoms of IBS have been described in medicine since 1849, using various names such as spastic (irritable) bowel syndrome or neurogenic mucous colitis. Today, this syndrome is defined as "intermittent abdominal pain or discomfort for 4 days in the last 2 months, in the absence of organic disease, followed by one or more of the following changes: changes in defecation rate, frequency, shape, and/or stool appearance, bloating, cramps and gas" (Nelms, 2010). Because of the nature of

*m.andreska@yahoo.com the syndrome, nutrition care plan should be provided to ensure better quality of life as well as favorable clinical outcome.

In this paper, a case of patient with IBS is presented and the role of an accredited dietitian in providing, expert dietary advice for management of IBS symptoms and nutritional needs is emphasized.

\section{Case presentation}

A 33-year-old woman was diagnosed with IBS. She had intermittent episodes of diarrhea and constipation and complains of distension and abdominal pain. Her physician advised her to supplement her diet with fiber and psyllium. The patient did not want to replace white bread with whole wheat bread. She also refused to use fiber (psyllium) in the form of a dietary supplement because she was convinced that the fibers helped with constipation but worsened diarrhea. She considered adding yogurt to her diet to see if it would help. For breakfast, she usually took a glass of orange juice, two slices of white bread with peanut butter and coffee, for snack small portion of chips, for lunch hamburger and a diet coke, for afternoon snack a portion of crackers with cheese and a glass of white wine. The usual dinner consisted of chicken meat prepared in sauce with 
potatoes, broccoli, as dessert ice cream and coffee and before bedtime, milk and cookies or apples. Given the health problems associated with the intestines, the patient had symptoms of depression. Physician recommended pharmaco-therapy that included oral magnesium hydroxide, linaclotide or loperamide as needed, dicyclomine as needed, fluoxetine at recommended dose, and gabapentin as needed.

\section{Discussion}

Nutritional intervention in patients with IBS should be based on the current situation. General recommendations include taking small meals throughout the day as well as probiotics to regulate the intestinal flora and reduce bloating and gas. To detect foods that exacerbate symptoms it is recommended that the patient keep a diet diary. In the phase of predominant diarrhea, one recommends adequate fluid intake, primarily water and herbal tea, restriction of caffeine-containing beverages, restriction of foods containing insoluble fiber (whole grain bread, nuts, seeds), restriction of eating fresh and dried fruit to 3 servings per day, reduction of starch intake and sugar free foods (containing sorbitol and xylitol), consumption of probioticcontaining products (yogurt, fermented dairy products) and avoiding fat-containing foods (chips, burgers, fried foods and sweets) as they can worsen diarrhea. In the predominant constipation phase, dietary fiber intake is recommended. It improves constipation, but causes an increased amount of gas in the intestine, which causes pain, flatulence and cramps. Individual customization of the selection of cereals is also recommended and of the quantity that will suit personally. Intake of fiber rich foods should be gradual, because any sudden increase in fiber intake can worsen symptoms. Symptoms of constipation can be improved by combination of whole grains with fruits and vegetables. Oatmeal and linseed are good sources of soluble fiber, which help to soften the stool and ease the passage and can also help alleviate the symptoms of gas and bloating. Adequate fluid intake of at least 8 glasses of caffeine-free fluids per day (best water) has to be ensured.
Since the patient refused to replace the food that she was usually consuming, it was important to receive timely and accurate nutrition information in order to adopt and implement a diet plan and condition control. The patient was educated about the composition of the food and its benefits and the complications that it can make in its condition. Due to inadequate intake and selection of foods and liquids according to the patient's condition there was a risk of malnutrition. Thus, in the acute phase of the condition, the patient was advised to take an enteral nutrition that will help relieve the symptoms faster and achieve remission.

Considering that the condition significantly affected her mental state, consultation with a psychologist was also recommended in order to obtain a complete treatment that will improve the overall condition.

\section{Conclusion}

Dietary changes in patients with IBS syndrome should be implemented in order to alleviate symptoms and improve quality of life. Monitoring patient's progress is essential and a health professional with an expertise in dietetics can significantly help in dietary management, education and counseling of patients.

\section{References}

McKenzie, Y.A., Bowyer, R.K., Leach, H., Gulia, P., Horobin, J., O'Sullivan, N.A., Pettitt, C., Reeves, L.B., Seamark, L., Williams, M., Thompson, J., Lomer, M.C., 2016. British Dietetic Association systematic review and evidence-based practice guidelines for the dietary management of irritable bowel syndrome in adults. J. Hum. Nutr. Diet. 29, 549-575.

Nelms, M.N., 2010. Diseases of the lower gastrointestinal tract, second ed., in: Cossio, Y. (Ed.), Nutrition Therapy \& Pathophysiology. Cengage Learning, Belmont, pp. 376-436. 